\title{
Azithromycin versus Cephalexin for Simple Traumatic Wounds in the Emergency Department: A Randomised Trial
}

\author{
Hamed Basir Ghafouri ${ }^{1}$, Morteza Zare ${ }^{2}$, Azam Bazrafshan ${ }^{2}$, Abbas Edalatkhah ${ }^{3} \&$ Niloofar Abazarian ${ }^{1}$ \\ ${ }^{1}$ Emergency Medicine Management Research Center, Iran University of Medical Sciences, Tehran, Iran \\ ${ }^{2}$ Neuroscience Research Center, Institute of Neuropharmacology, Kerman University of Medical Sciences, \\ Kerman, Iran \\ ${ }^{3}$ Emergency Medicine Departments, Yazd University of Medical Sciences, Yazd, Iran \\ Correspondence: Niloofar Abazarian, Emergency Medicine Management Research Center, Iran University of \\ Medical Sciences, Tehran 7619813159, Iran. Tel: 98-34-3226-3855. E-mail: Abzarian.niloofar@gmail.com
}

Received: November 16, 2015

Accepted: December 20, 2015 Online Published: February 24, 2016

doi:10.5539/gjhs.v8n10p185

URL: http://dx.doi.org/10.5539/gjhs.v8n10p185

\begin{abstract}
Objective: The aim of this study was to investigate efficacy of azithromycin versus cephalexin for infection prophylaxisis in patients with simple traumatic wounds managed at emergency department.

Method: This randomized controlled trial compared short-course therapy of once-daily azithromycin (500 $\mathrm{mg}$ before the wound repair followed by $250 \mathrm{mg}$ /day for 5 days) with cephalexin (1000 $\mathrm{mg}$ before wound repair followed by $250 \mathrm{mg}$ every 6 hours for 5 days) in the treatment of patients with simple traumatic wounds. A total of 366 patients were randomly selected for the study and 303 were evaluated for the final analysis.
\end{abstract}

Results: On completion of therapy, the rate of observed infection was $9.6 \%$ in the cephalexin group (15 patients, odds ratio $=0.77,95 \%$ confidence interval, 0.56 to 1.06 ) and $5.4 \%$ in the azithromycin group ( 8 patients, odds ratio $1.42,95 \%$ confidence interval, 0.80 to 2.52 ). Both treatment indicated similar prophylactic efficacy during the study $(\mathrm{P}=0.197)$.

Conclusion: Our study showed that Azithromycin as infection prophylaxis in simple traumatic wounds had the same effect as cephalexin but azithromycin is easier to use and more cost-effective compared to cephalexin.

Keywords: traumatic wounds, infection prophylaxis, azithromycin, cephalexin

\section{Introduction}

Simple traumatic wounds (including abrasions or lacerations) are commonly encountered in the emergency departments with the annual prevalence of $8 \%$ of trauma-related injuries (Cassell et al., 2005; Zehtabchi et al., 2012). The standard wound care protocol for managing these injuries usually includes vigorous wound irrigation; debridement and removal of foreign bodies to prevent the infection (Austrailian Wound Management Association, 2010; Zehtabchi et al., 2012; Berwald et al., 2014). Prophylactic antibiotics are also recommended for some traumatic injuries (animal bites, burns (Barajas-Nava et al., 2013) and open fractures (Petersen \& Waterman, 2011; Smit \& Boyle, 2014)) to reduce the rates of infections after wound repair. Surprisingly, little evidence supports the use of prophylactic antibiotics for simple traumatic injuries (that are not involving bone, tendon, vessel or nerve). Information on the use of prophylactic antibiotics in the management of simple traumatic wounds is limited in terms of methodological flaws and small sample sizes (Zehtabchi et al., 2012; Berwald et al., 2014). Although the efficacy and use of prophylactic antibiotics has been previously reviewed by authors (Cassell et al., 2005; Goldberg et al., 2012; Kreutzer et al., 2014), lack of reliable information underlines ongoing concerns on the growing use of prophylactic antibiotics in primary care.

While using prophylactic antibiotics in the management of simple traumatic wounds is controversial, the choice of the treatment is also challenging. Cephalexin is a beta-lactam antibiotic; It inhibits the third and last stage of bacterial cell wall synthesis, while, Azithromycin is a macrolide antibiotic which inhibits bacterial protein synthesis. Both Cephalexin and azithromycin are prescribed as prophylactic regimens for managing traumatic wounds in the primary care But information on the efficacy of these drugs has been remained limited (Kiani, 1991; Mallory, 1991). Although they are among the top prescription dispensed antibiotics in some countries 
(Government of Canada, 2014), their use and prescription dispensed patterns are not similar. According to recent evidence, the overall use of cephalexin has been dramatically reduced during the last decade (Government of Canada, 2014), in contrast azithromycin indicated the greatest increase in prescribing over this period.

Both cephalexin and azithromycin have been used as prophylactic antibiotics, yet the merit and the efficacy of these drugs in reducing the rate of infection after wound repair is unclear. The use of these antibiotics in the emergency departments seems to be based on clinical experiences or patient preferences rather than well documented, randomised trials, therefore more investigations are needed to address these debates. The present randomised, clinical trial aims to compare the prophylactic effect of Cephalexin and Azithromycin in the treatment of simple traumatic injuries in emergency departments.

\section{Methods}

\subsection{Study Design and Settings}

This study is a single-blind randomised trial, conducted at the Emergency Department (ED) of a university hospital of Iran University of Medical sciences during April 2012-April 2013. In this trial, the physician collecting clinical data were unaware of the assigned treatments.

\subsection{Selection of Participants}

Potential participants included ED patients ( $>16$ yrs) with simple traumatic wounds that needed prophylactic antibiotics based on physician's judgement and who did not meet the exclusion criteria composed of history of allergy to cephalosporines or macrolides; current using any antibiotics or immunosuppressive drugs or history of diabetes mellitus, any renal, liver or infectious disease or pregnancy. Simple traumatic wounds were defined as those wounds not involving deeper structures (bone, tendon, nerve or vessel) and are not penetrating and bite wounds. Using convenience sampling method, patients were selected to enrol in the study. After enrolment, included patients were randomly allocated to two groups (A and B) using random numbers table.

Written consent was obtained and assessments were conducted by an ED physician. Ethical approval has been granted by the Iran University of Medical Sciences Research Ethics Committee and carried out in accordance with Declaration of Helsinki (1989). Trial was registered in Iranian Registry of Clinical Trials (IRCT) (IRCT2012071810017N3).

\subsection{Intervention}

In group A, Azithromycin capsule (500 mg, Loghman Inc., Tehran, Iran) was administered before the wound repair. After repair, the patients received azithromycin $250 \mathrm{mg}$ once daily for 4 days (Drugsite Trust 2015). In the group B, the patients received $1000 \mathrm{mg}$ oral Cephalexin capsule (250 mg, Loghman Inc., Tehran, Iran) before the wound repair. Then, they were required to use Cephalexin $250 \mathrm{mg}$ every 6 hours for 5 days (Drugsite Trust 2015). Dosage of these two antibiotics was prescribed based on dosage recommended by most of references for treating skin infection. All of the patients were required to visit ED physician for removing their sutures during 5 days after their first ED visit. Patients were required to refer to ED physician or call the researcher in case of occurrence of any signs or symptoms of wound infection during their wound healing period (the day after receiving treatment until removing the sutures).

Outcome assessments were made by a blinded ED physician at the time of sutures removal.

\subsection{Outcome Measures}

Primary outcome measure was the difference in wound infection rates between two studied groups. Wound evaluations were based on assessing the signs (cellulitis $>1 \mathrm{~cm}$, lymphangitis, presence of discharge, presence of necrotic tissue and abscess) and symptoms (pain, dryness and itching) of wound infection and presence/absence of wound dehiscence. Wound infection was defined as the presence of any mentioned signs. Secondary outcome measures were the occurrence of skin irritation and wound dehiscence.

\subsection{Primary Data Analysis}

Assuming a baseline incidence of wound infections of $15 \%$ in repaired wounds in the emergency room, to detect a $1 \%$ or greater difference in our primary outcome between the two studied groups with an $\alpha$ of 0.05 and a $\beta$ of 0.2 ( $80 \%$ power), 250 patients were required for each group.

The primary outcome of this study was the presence or absence of a significant wound infection on the follow-up assessment. A wound infection was considered to be significant if the follow-up physician's impression was that there was a wound infection requiring supplemental antibiotics.

All data were analyzed with Stata software (version 12.0). Demographic and clinical data were presented 
descriptively as frequencies and means with SDs where appropriate. Chi square was used to compare the frequency of observed wound infection in the cephalexin and azithromycin groups. The significance level was 0.05 for two-sided tests.

\section{Results}

During the study period, 366 patients were screened and finally enrolled. Of those patients, 303 patients were evaluated for the final analysis. (Figure 1)

There were no differences in the baseline characteristics of the cephalexin and azithromycin groups (Table 1). Men constituted $84.5 \%$ of the study participants. The sites of wounds were head \& neck in $46 \%$ of patients, upper extremities in $33 \%$ of patients, lower extremities in $17 \%$ of patients' and truck in $4 \%$ of the patients. Wounds were typically treated on average 1-6 hours after the event (90\%). The average depth and length of traumatic wounds were similar in both groups ( $\mathrm{p}=0.287, \mathrm{p}=0.760$ accordingly).

The rate of observed infection was $9.6 \%$ in the cephalexin group ( 15 patients, odds ratio $=0.77,95 \%$ confidence interval, 0.56 to 1.06 ) and $5.4 \%$ in the azithromycin group ( 8 patients, odds ratio $1.42,95 \%$ confidence interval, 0.80 to 2.52$)$. The difference in infection rates was not statistically significant between these groups $(\mathrm{P}=0.197)$. (Table 2).

Table 1. Demographic characteristics of the study patients and baseline characteristics of the wounds

\begin{tabular}{|c|c|c|c|c|}
\hline \multicolumn{2}{|c|}{ Patients and Wound Characteristics } & \multirow{2}{*}{$\begin{array}{l}\begin{array}{l}\text { Cephalexin } \\
(n=156)\end{array} \\
130(83.3)\end{array}$} & \multirow{2}{*}{$\begin{array}{l}\text { Azithromycin } \\
(\mathrm{n}=147)\end{array}$} & \multirow{3}{*}{$\begin{array}{l}\mathrm{P} \text { value } \\
\mathrm{P}=0.567\end{array}$} \\
\hline Gender $(0 /)$ & Male & & & \\
\hline vender (\%) & Female & $26(16.7)$ & $21(14.3)$ & \\
\hline \multirow[t]{2}{*}{ Age $(\mathrm{M} \pm \mathrm{SD})$ (years) } & & $\begin{array}{l}33.88 \pm 16.90 \\
(3-93 \text { years) }\end{array}$ & $\begin{array}{l}31.63 \pm 14.04 \\
(11-86 \text { years })\end{array}$ & $\mathrm{P}=0.208$ \\
\hline & Head \& neck & $62(39.7)$ & $77(52.4)$ & \multirow{4}{*}{$\mathrm{P}=0.096$} \\
\hline \multirow{3}{*}{ Wound Location (\%) } & Upper limb & $55(35.3)$ & $46(31.3)$ & \\
\hline & Lower limb & $31(19.9)$ & $21(14.3)$ & \\
\hline & Trunk & $8(5.1)$ & $3(2)$ & \\
\hline \multirow{2}{*}{ Wound Mechanism (\%) } & Sharp & $111(71.2)$ & $99(67.3)$ & \multirow{2}{*}{$\mathrm{P}=0.473$} \\
\hline & Blunt & $45(28.8)$ & $48(32.7)$ & \\
\hline \multicolumn{2}{|c|}{ Wound Length $(\mathrm{M} \pm \mathrm{SD})(\mathrm{Cm})$} & $\begin{array}{l}4.05 \pm 2.19 \\
(1-13.5 \mathrm{~cm})\end{array}$ & $\begin{array}{l}4.33 \pm 2.50 \\
(1-15 \mathrm{~cm})\end{array}$ & $\mathrm{P}=0.287$ \\
\hline \multicolumn{2}{|c|}{ Wound Depth $(\mathrm{M} \pm \mathrm{SD})(\mathrm{Cm})$} & $\begin{array}{l}0.629 \pm 0.369 \\
(0.2-2.5 \mathrm{~cm})\end{array}$ & $\begin{array}{l}0.616 \pm 0.419 \\
(0.2-4.0 \mathrm{~cm})\end{array}$ & $\mathrm{P}=0.760$ \\
\hline \multirow[t]{3}{*}{ Repair Time (\%) } & $<1 \mathrm{~h}$ & $11(7.1)$ & $7(4.8)$ & \multirow{3}{*}{$\mathrm{P}=0.656$} \\
\hline & $1-6 \mathrm{~h}$ & $140(89.7)$ & $134(91.2)$ & \\
\hline & $\geq 6 \mathrm{~h}$ & $5(3.2)$ & $6(4.1)$ & \\
\hline
\end{tabular}

Table 2. The rates of infection after wound repair for patients receiving cephalexin or azithromycin

\begin{tabular}{lllll}
\hline Outcome & & $\begin{array}{l}\text { Cephalexin } \\
(\mathrm{n}=156)\end{array}$ & $\begin{array}{l}\text { Azithromycin } \\
(\mathrm{n}=147)\end{array}$ & P value \\
\hline Infection (\%) & Yes & $15(9.6)$ & $8(5.4)$ & $\mathrm{P}=0.197$ \\
& No & $141(90.4)$ & $139(94.6)$ & \\
\hline
\end{tabular}




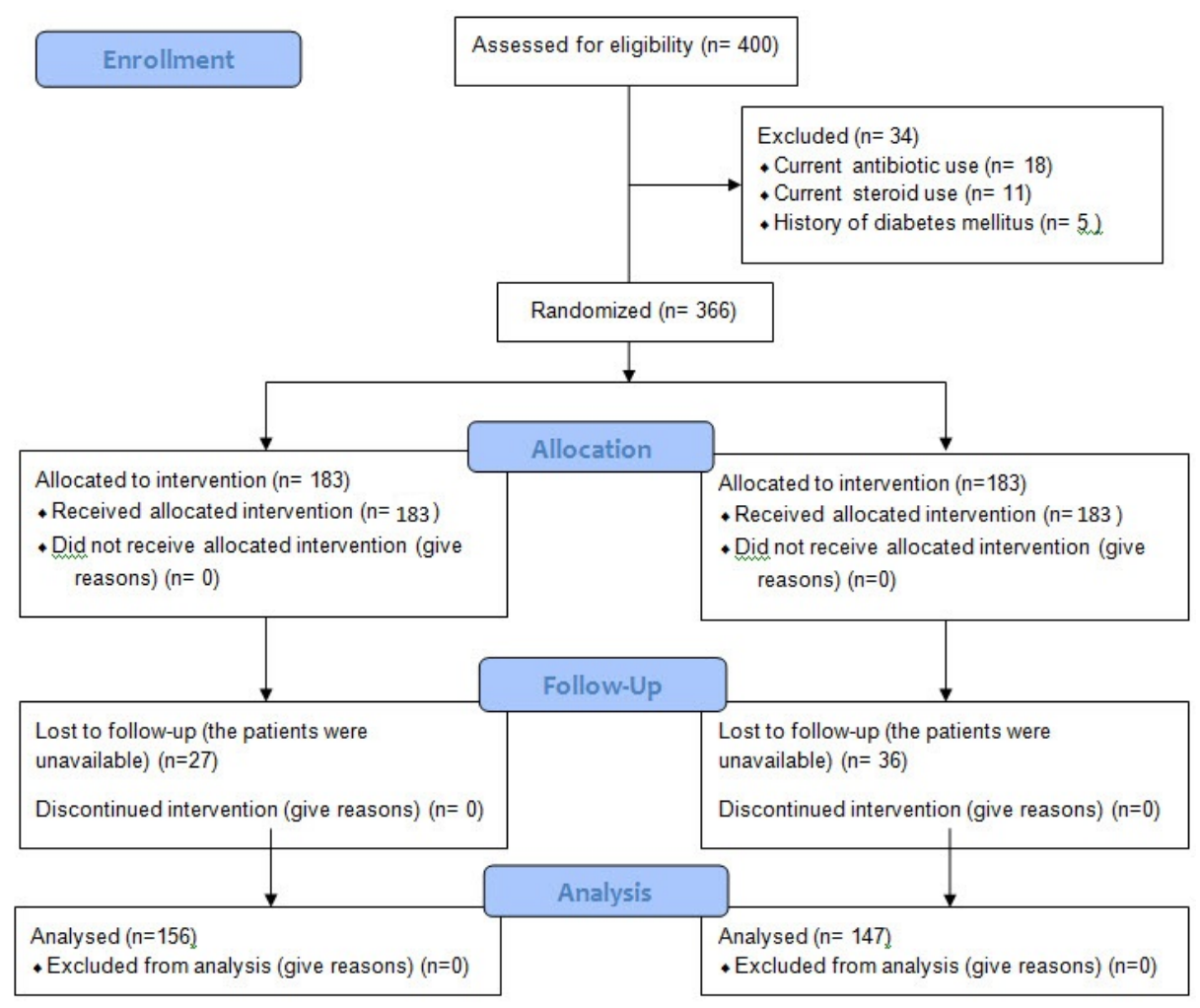

Figure 1. Flow diagram of CONSORT

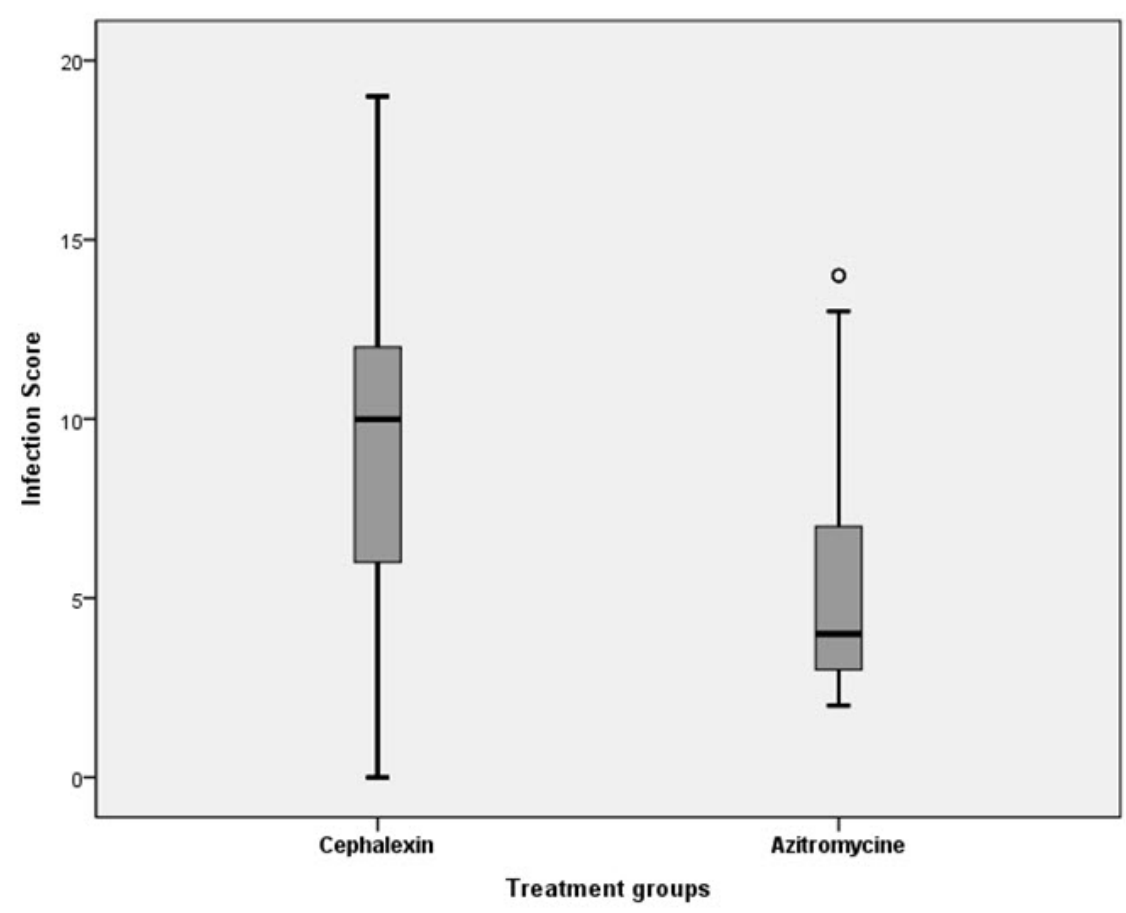

Figure 2. Distribution of infection score in cephalexin and azithromycin groups

\section{Discussion}

The present study evaluated the prophylactic efficacy of cephalexin versus azithromycin for simple traumatic wounds. Data from 303 patients were extracted and the results yielded a broad estimate of the efficacy of 
prophylactic antibiotics in ED settings so far.. Cephalexin and azithromycin indicated similar prophylactic efficacy in the management of simple traumatic wounds.

The efficacy of prophylactic antibiotics in the management of wounds has been previously investigated (Smith \& Wilson, 2013; Quinn et al., 2014) and the results confirmed the use of prophylactic antibiotics as standard care in reducing the rate of infection for some penetrating or open wounds, animal bites and surgeries (Cassell et al., 2005; Petersen \& Waterman, 2011; Goldberg et al., 2012; Barajas-Nava et al., 2013; Kreutzer et al., 2014; Smit $\&$ Boyle, 2014). However, little evidence has been found to support the use of prophylactic antibiotic for simple traumatic wounds (Pacioni et al., 2013; Berwald et al., 2014). Some studies investigated the efficacy of topical antibiotics in minor, uncomplicated traumatic injuries (Waterbrook et al., 2013). Although the results have been mixed, using topical antibiotics have resulted in lower infection rates than placebo or other comparison groups. Due to methodological flaws and lack of high quality evidence, it has been difficult to give clear recommendations for prophylactic antibiotic use.

This approach also allowed us to compare the efficacy of cephalexin and azithromycin as two commonly prescribed antibiotics in the primary care. Although little evidence supports the prophylactic efficacy of these antibiotics in the management of simple traumatic wounds, our findings revealed similar patterns for these antibiotics. Azithromycin have resulted in lower infection rates than cephalexin but the study failed to provide statistically significant differences between the two drugs. This finding suggests that a 5-day administration of once-daily azithromycin is as effective as a 5-day administration of cephalexin four times a day in the management of simple traumatic injuries; but azithromycin is easier to use and more cost-effective compared to cephalexin.

Azithromycin has been developed to treat a wide range of infections. As an acid-stable compound, azithromycin generate high and sustained concentrations in the tissues in contrast of the extremely low serum levels (Kiani, 1991; Mallory, 1991). Besides, azithromycin has been found to indicate rapid penetration and prolonged levels within the inflammatory fluid. This allows once daily dosage and a short 5-day course of treatment. Cephalexin, as a first generation cephalosporin, is particularly used for skin and soft tissue wound infections. However, the drug's concentration in the tissue is lower than azithromycin, resulted in higher doses with longer treatment duration.

Although findings from previous studies confirmed the similar clinical effectiveness of azithromycin and cephalexin for skin and skin structure infections (Kiani, 1991; Mallory, 1991; Girard et al., 1993), there have been much variations in their prescription patterns (Government of Canada, 2014). Azithromycin has been among the most commonly prescribed antimicrobial drugs for the last decade, in contrast the use of cephalexin has been reduced over this time frame. This may reflect the overall interest of patients and physicians toward prescription of antibiotics with lower doses or lower durations.

The wide spread use of antibiotics in primary care potentially increases the risk of adverse effects and antibiotic-associated complications (Samonis et al., 1993; Donskey et al., 2000; Kreutzer et al., 2014). Therefore, the use of most effective antibiotics with lower duration is essential. In this context, our findings suggest azithromycin as a preferred treatment for simple traumatic wound infections in the emergency settings.

Our study has several limitations. Firstly, only sutured lacerations have been included in the study. Surgical wounds, animal bites and burns were excluded from the study as they were not classified as traumatic injuries or required other clinical or surgical interventions. Secondly, this study had no placebo controlled arm. Therefore, it was impossible to estimate the rate of infections without an antibiotic. Despite these limitations, this study has several unique strengths. This is the first randomized controlled trial, ever conducted to examine the efficacy of prophylactic antibiotics for managing simple traumatic wounds. Besides, this study provides the best evidence for azithromycin in simple traumatic wound management.

\section{Conflict of Interest}

The authors declare that there is no conflict of interests regarding the publication of this paper.

\section{References}

Austrailian Wound Management Association. (2010). Standards for Wound Management. Austrailian Wound Management Association.

Barajas-Nava, L. A., Lopez-Alcalde, J., et al. (2013). Antibiotic prophylaxis for preventing burn wound infection. The Cochrane Library, (6). http://dx.doi.org/10.1002/14651858.cd008738.pub2

Berwald, N., Khan, F., et al. (2014). Antibiotic prophylaxis for ED patients with simple hand lacerations: A 
feasibility randomized controlled trial. The American journal of emergency medicine, 32(7), 768-771. http://dx.doi.org/10.1016/j.ajem.2014.03.043

Cassell, O. C. S., Burge, P., et al. (2005). Peri-operative antibiotics for superficial hand trauma (protocol): The Cochrane library. Chichester, UK: John Wiley \& Sons.

Donskey, C. J., Chowdhry, T. K., et al. (2000). Effect of antibiotic therapy on the density of vancomycin-resistant enterococci in the stool of colonized patients. New England Journal of Medicine, 343(26), 1925-1932. http://dx.doi.org/10.1056/NEJM200012283432604

Drugsite Trust. (2015a). Azithromycin Dosage. Retrieved January 13, 2015, from http://www.drugs.com/dosage/ azithromycin.html

Drugsite Trust. (2015b). Cephalexin Dosage. Retrieved January 13, 2015, from http://www.drugs.com/dosage/ cephalexin.html

Girard, D., Bergeron, J. M., et al. (1993). Comparison of azithromycin, roxithromycin, and cephalexin penetration kinetics in early and mature abscesses. Journal of Antimicrobial Chemotherapy, 31(suppl E), 17-28. http://dx.doi.org/10.1093/jac/31.suppl_E.17

Goldberg, S. R., Anand, R. J., et al. (2012). Prophylactic antibiotic use in penetrating abdominal trauma: An Eastern Association for the Surgery of Trauma practice management guideline. Journal of Trauma and Acute Care Surgery, 73(5), S321-S325. http://dx.doi.org/10.1097/TA.0b013e3182701902

Government of Canada. (2014). Human Antimicrobial Drug Use Report 2012/2013. Guelph, Ontario.

Kiani, R. (1991). Double-blind, double-dummy comparison of azithromycin and cephalexin in the treatment of skin and skin structure infections. European Journal of Clinical Microbiology and infectious diseases, 10(10), 880-884. http://dx.doi.org/10.1007/BF01975848

Kreutzer, K., Storck, K., \& Weitz, J. (2014). Current evidence regarding prophylactic antibiotics in head and neck and maxillofacial surgery. BioMed research international 2014. http://dx.doi.org/10.1155/2014/8 79437

Mallory, S. B. (1991). Azithromycin compared with cephalexin in the treatment of skin and skin structure infections. The American journal of medicine, 91(3), S36-S39. http://dx.doi.org/10.1016/0002-9343 (91) $90400-\mathrm{R}$

Pacioni, C., Amadori, L., Hijazi Raed, M. S., Tosoni, F., \& Ricevuti, G. (2013). Use of Antibiotics for Epidermal Wounds in Emergency Room: Correct Prophylaxis and Right Choice of the Treatment. The Open Emergency Medicine Journal, 5(suppl 1), 25-28. http://dx.doi.org/10.2174/1876542401305010025

Petersen, K., \& Waterman, P. (2011). Prophylaxis and treatment of infections associated with penetrating traumatic injury. Expert Review of Anticancer Therapy, 9(1), 81-96. http://dx.doi.org/10.1586/eri.10.155

Quinn, J. V., Polevoi, S. K., \& Kohn, M. A. (2014). Traumatic lacerations: What are the risks for infection and has the golden period of laceration care disappeared? Emergency Medicine Journal, 31(2), 96-100. http://dx.doi.org/10.1136/emermed-2012-202143

Samonis, G., Gikas, A., et al. (1993). Prospective evaluation of effects of broad-spectrum antibiotics on gastrointestinal yeast colonization of humans. Antimicrobial agents and chemotherapy, 37(1), 51-53. http://dx.doi.org/10.1128/AAC.37.1.51

Smit, L., \& Boyle, M. (2014). Antibiotic prophylaxis in pre-hospital trauma care: A review of the literature. Australasian Journal of Paramedicine, 11(5).

Smith, B. C., \& Wilson, A. H. (2013). Topical versus injectable analgesics in simple laceration repair: An integrative review. The Journal for Nurse Practitioners, 9(6), 374-380. http://dx.doi.org/10.1016/j.nurpra. 2013.03.004

Waterbrook, A. L., Hiller, K., et al. (2013). Do topical antibiotics help prevent infection in minor traumatic uncomplicated soft tissue wounds? Annals of emergency medicine, 61(1), 86-88. http://dx.doi.org/10.1016/j. annemergmed.2012.08.002

Zehtabchi, S., Yadav, K., et al. (2012). Prophylactic antibiotics for simple hand lacerations: Time for a clinical trial? Injury, 43(9), 1497-1501. http://dx.doi.org/10.1016/j.injury.2011.05.001 


\section{Copyrights}

Copyright for this article is retained by the author(s), with first publication rights granted to the journal.

This is an open-access article distributed under the terms and conditions of the Creative Commons Attribution license (http://creativecommons.org/licenses/by/3.0/). 